\title{
Barra de cereais com adição de farinha de resíduos de rabanete: caracterização físico-química e sensorial entre crianças
}

\section{Cereal bars with addition of radish waste flour: physicochemical and sensory characterization among children}

Barra de cereal con harina de residuos de rábano añadida: caracterización fisicoquímica y sensorial entre niñose

Larissa Gabriela da Silva Andrade ${ }^{1}$ Tainá da Silva Fleming de Almeida ${ }^{2}$ Elisvânia Freitas dos Santos ${ }^{3}$ Daiana Novello ${ }^{4}$

${ }^{1}$ Graduanda em Nutrição, Universidade Estadual do Centro-Oeste (UNICENTRO), Campus CEDETEG. E-mail: larisandrade2000@outlook.com, ORCID: http://orcid.org/0000-0001-6858-5197

${ }^{2}$ Mestranda do Programa de Pós-graduação em Biotecnologia pela Universidade Federal de Mato Grosso do Sul (UFMS). Graduada em Nutrição pela UFMS. E-mail: tainaafleming@gmail.com, ORCID: http://orcid.org/0000-0003-1873-8452

${ }^{3}$ Doutora em Cirurgia Experimental pela Universidade Estadual de Campinas (UNICAMP). Mestre em Alimentos e Nutrição pela UNICAMP. Especialista em Nutrição Humana e Saúde pela Universidade Federal de Lavras (UFLA). Graduada em Nutrição pelo Centro Universitário de Votuporanga. Professora do Curso de Nutrição e do Programa de Pós-Graduação em Biotecnologia da Faculdade de Ciências Farmacêuticas, Alimentos e Nutrição (FACFAN), da Universidade Federal de Mato Grosso do Sul (UFMS), Campo Grande, MS, Brasil. E-mail: elisvania@gmail.com, ORCID: http://orcid.org/0000-0002-1528-6035

${ }^{4}$ Doutora em Tecnologia de Alimentos pela Universidade Estadual de Campinas (UNICAMP). Mestre em Ciências Veterinárias pela Universidade Federal do Paraná (UFPR). Especialista em Ciências Sensoriais e Estudos com Consumidores pela About Solution. Graduada em Nutrição pela Universidade Regional do Noroeste do Estado do Rio Grande do Sul (UNIJUÍ). Docente do Curso de Nutrição e do Programa de Pós-Graduação Interdisciplinar em Desenvolvimento Comunitário da Universidade Estadual do Centro-Oeste (UNICENTRO), Campus CEDETEG, Guarapuava, PR, Brasil. E-mail: nutridai@hotmail.com, ORCID: http://orcid.org/0000-0003-0762-5292 
Resumo: Objetivou-se avaliar a aceitabilidade sensorial de barra de cereais adicionada de farinha de resíduos de rabanete (FRR) e determinar a composição físico-química da formulação padrão e daquela contendo maior teor de FRR com aceitação semelhante ao padrão. Foram elaboradas cinco formulações de barra de cereais: F1 (0\% de FRR) e as demais adicionadas de 2,5\% (F2), 5\% (F3), 7,5\% (F4) e 10\% (F5) de FRR. Maiores notas foram verificadas para F1, F2 e F3, enquanto F4 e F5 foram menos aceitas. F3 foi a amostra com maior teor de FRR e aceitação sensorial similar ao padrão. Maiores teores de umidade, cinzas, proteína, lipídio e fibras e menores de carboidrato e calorias foram verificados em F3. Um nível de adição de até $5 \%$ de FRR em barra de cereais melhora o perfil nutricional do produto. Além disso, apresenta aceitabilidade semelhante ao produto padrão, com boas expectativas de comercialização.

Palavras-chave: subprodutos; hortaliça; resíduo alimentar.

\begin{abstract}
The objective of this study was to evaluate the sensorial acceptability of a cereal bar added with radish waste flour (RWF) and to determine the physicochemical composition of the standard formulation and that containing the highest RWF content with acceptance similar to the standard. Five cereal bar formulations were prepared: F1 (0\% RWF) and the others added 2.5\% (F2), 5\% (F3), 7.5\% (F4) and 10\% (F5) RWF. Higher scores were verified for F1, F2 and F3, while F4 and F5 were less accepted. F3 was the sample with the highest RWF content and sensory acceptance similar to the standard. Higher levels of moisture, ash, protein, lipid and fiber and lower levels of carbohydrate and calories were found in F3. An addition level of up to $5 \%$ of RWF in cereal bar improves the nutritional profile of the product. In addition, it presents acceptability similar to the standard product, with good commercialization expectations.
\end{abstract}

Keywords: byproducts; vegetable; food waste.

Resumen: El objetivo de este estudio fue evaluar la aceptabilidad sensorial de una barra de cereal agregada con harina de residuos de rábano (HRR) y determinar la composición fisicoquímica de la formulación estándar y aquella que contenga el mayor contenido de HRR con aceptación similar a la estándar. Se prepararon cinco formulaciones de barras de cereal: F1 (0\% HRR) y las otras agregaron $2.5 \%$ (F2), 5\% (F3), 7.5\% (F4) y 10\% (F5) HRR. Se verificaron puntuaciones más altas para F1, F2 y F3, mientras que F4 y F5 fueron menos aceptadas. F3 fue la muestra con mayor contenido de HRR y aceptación sensorial similar al estándar. En F3 se encontraron niveles más altos de humedad, cenizas, proteína, lípido y fibra y niveles más bajos de carbohidrato y calorías. Un nivel de adición de hasta un $5 \%$ de HRR en la barra de cereal mejora el perfil nutricional del producto. Además, presenta una aceptabilidad similar al producto estándar, con buenas expectativas de comercialización.

Palabras clave: subproductos; hortaliza; desperdicio de alimentos. 
Barra de cereais com adição de farinha de resíduos de rabanete: caracterização físico-química e sensorial entre crianças

\section{INTRODUÇÃO}

O desperdício de alimentos é considerado um problema de saúde pública. Os alimentos que apresentam maior taxa de desperdício são as frutas e as hortaliças. As fases de produção, processamento, distribuição, armazenamento e consumo são aquelas em que mais ocorrem perdas. Entretanto também se verifica um elevado desperdício na fase de preparo dos alimentos, sendo que as cascas, as folhas, os talos e as sementes são os subprodutos com maior rejeito. Em nível mundial, estima-se que cerca de um terço de todo o alimento produzido é perdido ou desperdiçado, o que corresponde a bilhões de toneladas/ano de resíduos descartados no meio ambiente.

O uso de subprodutos derivados de hortaliças como ingrediente em preparações alimentícias já é considerado uma alternativa viável para auxiliar na redução do desperdício de alimentos. Isso porque as cascas, sementes, folhas e talos contêm um elevado teor nutricional, muitas vezes superior à polpa. Além de melhorar o valor nutritivo, a adição de resíduos alimentares em produtos pode agregar valor de mercado e colaborar para a redução do lixo ambiental. Pesquisas já comprovaram a viabilidade do uso de farinha de resíduos de hortaliças em barra de cereais, cookie e pão. Os resultados demonstraram uma boa aceitabilidade sensorial e melhora no perfil nutricional dos produtos, especialmente no aumento do teor de fibras. Contudo houve prejuízo na textura da massa, apresentando um aspecto quebradiço e coloração diferente do produto normalmente consumido. Além disso, níveis muito altos dos resíduos promoveram uma redução das notas sensoriais, especialmente para o sabor.

O rabanete (Raphanus sativus) é uma planta herbácea, de porte pequeno, com formato arredondado e alongado, possui casca de coloração predominantemente avermelhada e polpa branca. A produção mundial da hortaliça é estimada em sete milhões de toneladas por ano, sendo o Japão um dos maiores produtores. No Brasil, o consumo da hortaliça ainda é baixo. A maior produção do rabanete ocorre a partir do mês de maio, devido às temperaturas mais baixas, geralmente entre 8 으 e 20 으. Em 2016, o rabanete ficou entre os 20 produtos mais vendidos do setor de hortaliças, aproximadamente 1.500 toneladas. Além do sabor levemente picante, o rabanete contém elevado teor de fibra alimentar, vitamina $\mathrm{C}$, folato e mine- 
rais como cálcio, ferro e magnésio. As folhas e os talos do rabanete são, quase sempre, descartados, apesar de conterem altos teores de minerais. Dessa forma, demonstra-se o potencial de utilização de resíduos alimentícios no desenvolvimento de novos produtos, como os talos e as folhas de rabanete. Com isso, é possível colaborar para a oferta de alimentos mais saudáveis para a população, além de colaborar para a redução do lixo orgânico.

As barras de cereais fazem parte da categoria de cereais processados e apresentam uma elevada aceitação pelos mais diversos públicos. Esse fato se deve ao baixo custo e, especialmente, pela praticidade, já que é uma alternativa de fácil consumo e aquisição. Em alguns casos, a barra de cereais pode conter ingredientes menos favoráveis ao consumo, como, por exemplo, a farinha de trigo refinada. Nesse aspecto, essa matéria-prima poderia ser substituída por resíduos alimentícios de frutas e hortaliças que apresentem maior teor nutritivo. Assim, a barra de cereais torna-se um produto com elevado potencial tecnológico para adição de novos ingredientes, especialmente para ser oferecida a públicos mais vulneráveis como as crianças.

A faixa etária dos 7 a 10 anos é classificada como idade escolar. Nessa fase, os hábitos alimentares das crianças estão em constante desenvolvimento. Contudo elas estão expostas a fatores sociais, ambientais, familiares e culturais que podem influenciar no consumo de alimentos, o que, geralmente, perdura até a fase adulta. Atualmente, o público infantil apresenta uma elevada ingestão de alimentos com altos teores de açúcar, gordura e sódio. Além disso, o consumo de frutas e hortaliças está bem aquém da recomendação adequada de, no mínimo, cinco porções diárias. Esse tipo de escolha alimentar pode estar relacionada a diversas patologias crônicas não transmissíveis, como a obesidade, as doenças cardiovasculares e o câncer. Sabendo-se disso, o ambiente escolar torna-se favorável para o desenvolvimento de ações que possam corrigir a alimentação infantil. Um exemplo é a oferta de produtos alimentícios com um perfil nutricional mais favorável, os quais possam proporcionar às crianças um crescimento e desenvolvimento adequados. Nesse contexto, os objetivos do trabalho foram avaliar a aceitabilidade sensorial de barra de cereais adicionada de diferentes níveis de farinha de resíduos de rabanete (FRR) entre crianças e determinar a composição físico-química do produto tradicional e daquele com maior teor de FRR e aceitação semelhante ao padrão. 
Barra de cereais com adição de farinha de resíduos de rabanete: caracterização físico-química e sensorial entre crianças

\section{MATERIAL E MÉTODOS}

Os ingredientes foram adquiridos em supermercados do município de Guarapuava, Paraná (PR). Foram utilizados resíduos (talos e folhas) de rabanete (Raphanus sativus), com melhor aspecto visual, consistência firme, sem imperfeições e de coloração esverdeada. Trinta quilos de resíduos de rabanete foram higienizados em água corrente potável, sanitizados (mergulhados em solução de hipoclorito de sódio por 15 minutos) e novamente higienizados em água corrente. Os resíduos foram extraídos de forma manual e picados. Em seguida, foram submetidos à secagem em desidratador (Pardal ${ }^{\circ}$, Brasil) com circulação de $\operatorname{ar}\left(65^{\circ} \mathrm{C}\right)$ por 48 horas. Depois de secos, permaneceram em temperatura ambiente $\left(22^{\circ} \mathrm{O}\right)$ até total resfriamento. Os resíduos foram triturados em liquidificador doméstico (Mondial ${ }^{\circledR}$, Brasil) e passados em peneira com abertura de 32 mesh/Tyler (Bertel ${ }^{\circ}$, Brasil) até a obtenção da FRR, com rendimento de 2,5 kg.

Foram elaboradas cinco formulações de barra de cereais adicionadas de diferentes níveis de FRR: F1 (0\%-padrão), F2 (2,5\%), F3 (5\%), F4 (7,5\%) e F5 (10\%). Essas porcentagens foram definidas por meio de testes sensoriais preliminares realizados com o produto. Além da FRR, os ingredientes utilizados nas formulações foram: açúcar mascavo (25,65\%), farinha de trigo (F1: 15\%, F2: 12,5\%, F3: 10\%, F4: 7,5\%, F5: 5\%), linhaça dourada (12,70\%), amendoim torrado $(9,77 \%)$, farinha de aveia (9,53\%), manteiga $(8,55 \%)$, aveia em flocos $(6,60 \%)$, chia $(6,11 \%)$ e água $(6,11 \%)$. A barra de cereais foi preparada misturando-se todos os ingredientes manualmente até completa homogeneização. Cada formulação foi disposta em formas de alumínio retangular $(24 \mathrm{~cm} \times 10 \mathrm{~cm} \times 5 \mathrm{~cm}$ ) e assadas em forno semi-industrial (Venâncio ${ }^{\circ}$, Brasil), preaquecido a 200 C por 15 minutos. Após esse processo, permaneceram em repouso até atingirem a temperatura ambiente $(22 \circ \mathrm{C})$. As barras de cereais foram cortadas em tamanho aproximado de $4 \mathrm{~cm} \times 3$ $\mathrm{cm}$ e acondicionadas em recipientes plásticos hermeticamente fechados até o momento das análises.

Participaram da análise sensorial 68 julgadores não treinados, sendo crianças devidamente matriculadas em uma Escola Municipal de Guarapuava, $\mathrm{PR}$, de ambos os sexos, com idade entre 7 e 10 anos. Os produtos foram 
submetidos à análise sensorial em uma sala da escola. Cada julgamento sensorial foi feito individualmente, sendo que o provador foi orientado pelas pesquisadoras para o preenchimento das respostas. Foram avaliados os atributos de aparência, aroma, sabor, textura e cor, por meio de uma escala hedônica facial estruturada mista de 7 pontos, variando de 1 (super ruim) a 7 (super bom). Também, foram aplicadas questões de aceitação global e de intenção de compra analisadas com o uso de uma escala estruturada de 5 pontos (1 - desgostei muito/não compraria a 5 - gostei muito/compraria com certeza) (DUTCOSKY, 2013). Os julgadores receberam uma porção de cada amostra (aproximadamente $15 \mathrm{~g}$ ), em pratos brancos descartáveis, codificados com números de três dígitos, de forma casualizada e balanceada, acompanhada de um copo de água para limpeza do palato. As formulações foram oferecidas aos julgadores de forma monádica sequencial. O cálculo do IA foi realizado conforme a fórmula: $I A(\%)=A \times 100 / B$ (em que: $A=$ nota média obtida para o produto e $B=$ nota máxima dada ao produto) (TEIXEIRA; MEINERT; BARBETTA, 1987).

As seguintes análises físico-químicas foram realizadas em triplicata na FRR, na formulação padrão e naquela com maior nível de adição de FRR e com aceitação sensorial semelhante ao produto padrão: Umidade: determinada em estufa a $105^{\circ} \mathrm{C}$ até peso constante; Cinzas: analisadas em mufla $\left(550^{\circ} \mathrm{C}\right)$; Proteína: avaliadas através do teor de nitrogênio total da amostra, pelo método Kjeldahl, determinado ao nível semimicro. Utilizou-se o fator de conversão de nitrogênio para proteína de 6,25; fibra alimentar total e insolúvel: determinadas por método enzimático. 0 teor de fibra alimentar solúvel foi calculado pela diferença dos resultados de fibra alimentar total e insolúvel (AOAC, 2011); lipídio: utilizou-se o método de extração a frio (BLIGH; DYER, 1959); carboidrato: avaliados por cálculo teórico (por diferença) nos resultados das triplicatas, conforme a fórmula: $\%$ carboidrato = $100-(\%$ umidade + \% proteína + \% lipídio + \% cinzas + \% fibra); valor calórico total (kcal): calculado utilizando-se os seguintes valores: lipídio $\left(8,37 \mathrm{kcal} \mathrm{g}^{-1}\right)$, proteína (3,87 kcal g-1) e carboidrato (4,11 kcal g-1) (MERRILL; WATT, 1973). Valor Diário de Referência (VD): calculado em relação a $25 \mathrm{~g}$ de barra de cereais, com base nos valores médios diários preconizados para crianças (7 a 10 anos) (IOM, 2005), resultando em: 1.933,5 kcal/dia, $130 \mathrm{~g} / \mathrm{dia}$ de carboidrato, 26,5 g/dia de proteína e 26,75 g/dia de fibra alimentar. 
Os dados foram analisados com auxílio do software Statgraphics Plus ${ }^{\bullet}$, versão 5.1, por meio da análise de variância (ANOVA). A comparação de médias foi realizada pelos testes de Tukey e t de Student, avaliados com nível de 5\% de significância.

Este trabalho foi aprovado pelo Comitê de Ética em Pesquisa da UNICENTRO, parecer número n. 2.451.570/2017. Como critérios de exclusão, foram considerados os seguintes fatores: possuir alergia a algum ingrediente utilizado na elaboração da barra de cereais ou não entregar o Termo de Consentimento Livre e Esclarecido (TCLE) assinado pelo responsável legal.

\section{RESULTADOS E DISCUSSÃO}

Os resultados da análise sensorial da barra de cereais adicionada de diferentes níveis FRR estão descritos na Tabela 1.

Tabela 1 - Escores sensoriais (média desvio-padrão) e Índice de Aceitabilidade (IA) da barra de cereais com adição de diferentes níveis de farinha de resíduos de rabanete (FRR)

\begin{tabular}{lccccc}
\hline Parâmetros & F1 & F2 & F3 & F4 & F5 \\
\hline Aparência & $5,22 \pm 1,64^{\mathrm{a}}$ & $5,01 \pm 1,31^{\mathrm{a}}$ & $4,94 \pm 1,72^{\mathrm{a}}$ & $3,47 \pm 2,03^{\mathrm{b}}$ & $3,46 \pm 1,85^{\mathrm{b}}$ \\
IA (\%) & 74,57 & 71,57 & 70,57 & 49,57 & 49,42 \\
Aroma & $5,24 \pm 1,46^{\mathrm{a}}$ & $5,03 \pm 1,24^{\mathrm{a}}$ & $4,93 \pm 1,53^{\mathrm{a}}$ & $3,89 \pm 1,78^{\mathrm{b}}$ & $3,16 \pm 2,07^{\mathrm{b}}$ \\
IA (\%) & 74,86 & 71,86 & 70,43 & 55,57 & 45,14 \\
Sabor & $4,98 \pm 1,54^{\mathrm{a}}$ & $5,08 \pm 1,80^{\mathrm{a}}$ & $4,93 \pm 1,87^{\mathrm{a}}$ & $3,74 \pm 2,11^{\mathrm{b}}$ & $3,46 \pm 2,00^{\mathrm{b}}$ \\
IA (\%) & 71,14 & 72,57 & 70,43 & 53,43 & 49,43 \\
Textura & $4,95 \pm 1,66^{\mathrm{a}}$ & $4,93 \pm 1,77^{\mathrm{a}}$ & $4,91 \pm 1,65^{\mathrm{a}}$ & $3,84 \pm 1,93^{\mathrm{b}}$ & $3,12 \pm 1,82^{\mathrm{b}}$ \\
IA (\%) & 70,71 & 70,43 & 70,14 & 54,86 & 44,57 \\
Cor & $5,1 \pm 1,73^{\mathrm{a}}$ & $5,14 \pm 1,37^{\mathrm{a}}$ & $4,91 \pm 1,52^{\mathrm{a}}$ & $4,25 \pm 1,98^{\mathrm{b}}$ & $3,63 \pm 1,81^{\mathrm{b}}$ \\
IA (\%) & 72,86 & 73,43 & 70,14 & 60,71 & 51,86 \\
Aceitação global & $3,92 \pm 1,00^{\mathrm{a}}$ & $3,76 \pm 1,09^{\mathrm{a}}$ & $3,83 \pm 1,15^{\mathrm{a}}$ & $3,13 \pm 1,41^{\mathrm{b}}$ & $3,09 \pm 1,50^{\mathrm{b}}$ \\
IA (\%) & 78,40 & 75,20 & 76,60 & 62,60 & 61,80 \\
Intenção de compra & $3,82 \pm 1,22^{\mathrm{a}}$ & $3,58 \pm 1,17^{\mathrm{a}}$ & $3,69 \pm 1,46^{\mathrm{a}}$ & $2,78 \pm 1,51^{\mathrm{b}}$ & $2,97 \pm 1,40^{\mathrm{b}}$ \\
\hline
\end{tabular}

* Letras distintas na linha indicam diferença significativa pelo teste de Tukey $(p<0,05)$; Adição de FRR: F1: 0\%, F2: 2,5\%, F3: 5\%, F4: 7,5\%, F5: $10 \%$.

Fonte: Autoria própria (2020). 
A adição de níveis mais elevados de FRR (7,5\% e 10\%) reduziu a aceitação da barra de cereais em todos os parâmetros avaliados. As amostras F1, F2 e F3 tiveram notas de aceitação superiores às F4 e F5, sem diferença significativa entre si $(p>0,05)$. Resultados similares foram verificados após a adição de farinha de casca de chuchu (0\% a 27\%) em barra de cereal (CRISTO et al., 2015) e de farinha de casca de beterraba (0\% a 25\%) em cookie (TEIXEIRA et al., 2017). Os vegetais são compostos por substâncias fenólicas que têm um anel aromático contendo um ou mais grupos hidroxila. Em grandes quantidades, podem proporcionar sabor amargo, fato que prejudica a aceitação dos produtos. No caso dos resíduos do rabanete, os elevados teores de ácido cítrico e de compostos fenólicos podem promover um sabor ácido e adstringente, o que reduz as notas para a barra de cereais contendo os maiores teores de FRR.

Durante a elaboração do produto, verificou-se um aspecto mais quebradiço para as formulações F4 e F5 após a cocção. Esse efeito ocorre devido ao elevado teor de fibras da FRR. As fibras apresentam capacidade higroscópica, o que aumenta a absorção de água. Isso pode modificar o comportamento estrutural da massa, diminuindo a elasticidade e, consequentemente, afetando a qualidade final do produto. Além da alteração na textura, maiores concentrações de FRR promoveram uma cor esverdeada à barra de cereal, já que os resíduos do rabanete apresentavam uma cor verde/amarronzada (Figura 1). Índices de aceitabilidade maiores que 70\% foram verificados para as formulações F1, F2 e F3, classificando-as com boa aceitabilidade (TEIXEIRA; MEINERT; BARBETTA, 1987).

Figura 1 - Farinha de Resíduos de Rabanete

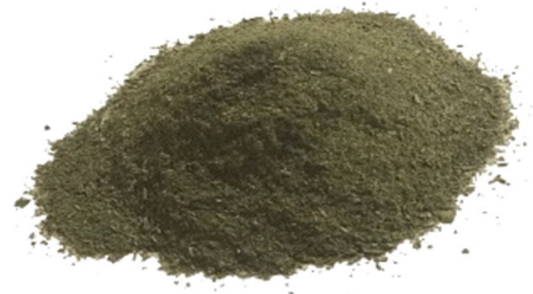

Fonte: Autoria própria. 
Barra de cereais com adição de farinha de resíduos de rabanete: caracterização físico-química e sensorial entre crianças

Figura 2 - Formulações de barra de cereais adicionadas de diferentes níveis de farinha de resíduos de rabanete: F1 (0\%), F2 (2,5\%), F3 (5\%), F4 (7,5\%) e F5 (10\%)

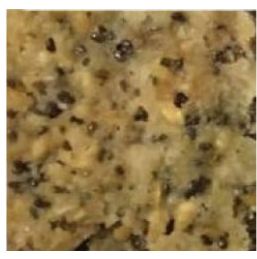

F1

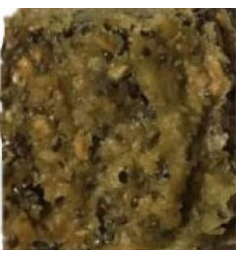

F2

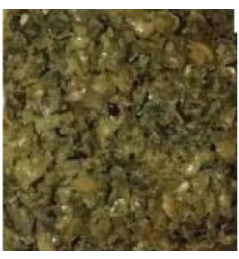

F3

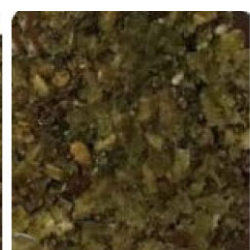

F4

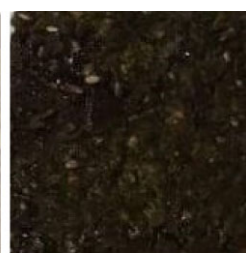

F5

\section{FORMULAÇÕES}

Fonte: Autoria própria.

Considerando que a amostra F3 foi aquela com aceitação sensorial similar à padrão em todas as avaliações (Tabela 1), ela foi selecionada para fins de comparação físico-química juntamente da formulação padrão (F1) (Tabela 2).

Tabela 2 - Composição físico-química média ( \pm desvio-padrão) da farinha de resíduos de rabanete (FRR), da barra de cereais padrão (F1) e com adição de $5 \%$ (F3) de FRR

\begin{tabular}{|c|c|c|c|c|c|}
\hline Parâmetro & FRR & F1 & VD (\%)* & F3 & VD (\%)* \\
\hline Umidade (g $100 \mathrm{~g}^{-1}$ ) & $4,38 \pm 0,04$ & $10,35 \pm 0,02^{b}$ & ND & $13,98 \pm 0,03^{\mathrm{a}}$ & ND \\
\hline Cinzas (g $100 \mathrm{~g}^{-1}$ ) & $15,89 \pm 0,05$ & $1,50 \pm 0,04^{b}$ & ND & $1,63 \pm 0,02^{a}$ & ND \\
\hline Proteína (g $100 \mathrm{~g}^{-1}$ ) & $28,41 \pm 0,07$ & $10,31 \pm 0,03^{b}$ & 9,73 & $11,70 \pm 0,09^{a}$ & 11,04 \\
\hline Lipídio (g 100 g $^{-1}$ ) & $4,68 \pm 0,09$ & $15,89 \pm 0,05^{b}$ & ND & $18,33 \pm 0,06^{\mathrm{a}}$ & ND \\
\hline Carboidrato $\left(\mathrm{g} 100 \mathrm{~g}^{-1}\right)^{* *}$ & $46,64 \pm 0,56$ & $61,96 \pm 0,13^{\mathrm{a}}$ & 11,91 & $54,36 \pm 0,55^{b}$ & 10,45 \\
\hline 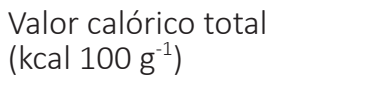 & $340,78 \pm 0,63$ & $427,51 \pm 0,78^{a}$ & 5,53 & $422,11 \pm 0,87^{b}$ & 5,46 \\
\hline Fibra solúvel (g $\left.100 \mathrm{~g}^{-1}\right)^{* * *}$ & $7,41 \pm 0,56$ & $2,47 \pm 0,10^{b}$ & ND & $2,84 \pm 0,12^{a}$ & ND \\
\hline Fibra insolúvel $\left(\mathrm{g} 100 \mathrm{~g}^{-1}\right)^{* * *}$ & $26,95 \pm 0,25$ & $4,45 \pm 0,15^{b}$ & ND & $5,68 \pm 0,25^{\mathrm{a}}$ & ND \\
\hline 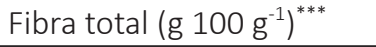 & $34,36 \pm 0,30$ & $6,91 \pm 0,22^{b}$ & 6,46 & $8,51 \pm 0,25^{a}$ & 7,95 \\
\hline
\end{tabular}

Letras distintas na linha indicam diferença significativa pelo teste de t de Student $(p<0,05)$; *Valor Diário de Referência (VD): nutrientes avaliados pela média da DRI (IOM, 2005), com base numa dieta de $1.933,5 \mathrm{kcal}^{\mathrm{dia}}{ }^{-1}$ e porção média de 25 gramas de produto; valores apresentados em base úmida; ${ }^{* *}$ inclui fibra alimentar; ${ }^{* * *}$ fibra alimentar; ND: não disponível Fonte: Autoria própria. 
Fatores relacionados ao plantio, como a qualidade do solo, o período do ano e as condições climáticas, além de aspectos de preparo da farinha, como as partes utilizadas, o tempo de secagem e a temperatura, podem influenciar no teor nutricional das hortaliças. A FRR apresenta elevada concentração de minerais, principalmente o cálcio, potássio e fósforo. O teor de umidade da FRR está adequado à legislação vigente, que recomenda até 15\% para farinhas (BRASIL, 2005).

A amostra F3 apresentou o maior teor de umidade $(p<0,05)$, já que as fibras presentes na FRR retêm água no produto, devido a sua elevada higroscopicidade. Dessa forma, pode haver uma redução da crocância do produto, provocando o amolecimento e rejeição pelos consumidores. A adição de FRR elevou o teor de cinzas em F3, já que apresenta um maior

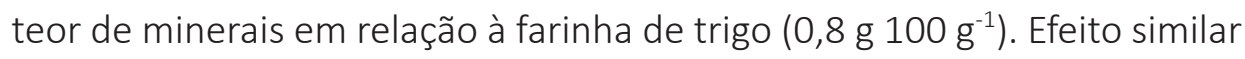
foi observado para os teores de fibras solúvel, insolúvel e total, que também foram maiores $(p<0,05)$ na formulação $F 3$, considerando que a FRR contém elevado conteúdo de fibras comparado à farinha de trigo $\left(2,30 \mathrm{~g} 100 \mathrm{~g}^{-1}\right)$ (UNICAMP, 2011).

Maiores teores de proteína e lipídio e menores de carboidrato e calorias $(p<0,05)$ foram observados para F3. Efeito que ocorre porque a farinha

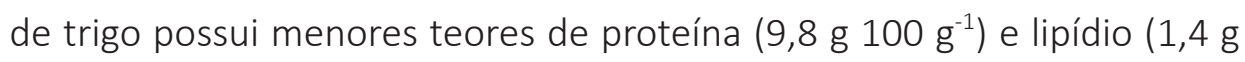

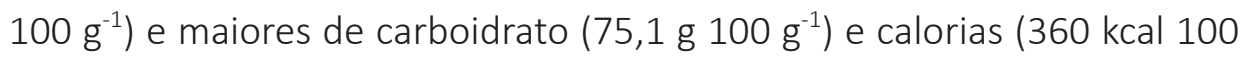
$\mathrm{g}^{-1}$ ) (UNICAMP, 2011) comparada à FRR. Esses resultados corroboram com Cristo et al. (2015), que estudaram barra de cereais com adição de farinha de casca de chuchu (0\%, 6,75\%, 13,5\%, 20,25\%, 27\%). A formulação contendo $5 \%$ de FRR (F3) apresentou um aumento de $23 \%$ em relação ao teor de fibra alimentar total, quando comparada à F1. Nesse aspecto, a amostra F3 pode ser considerada com alto teor de fibra alimentar, já que possui uma quantidade mínima de $6 \%$ de fibra em sua composição (BRASIL, 2012). Os resultados encontrados nesta pesquisa são positivos e podem favorecer um consumo alimentar mais saudável entre crianças, especialmente em relação à fibra. Nesse aspecto, há evidências de que a ingestão adequada de fibras pode reduzir o risco de doenças crônicas não transmissíveis, como a hipertensão arterial, a diabetes mellitus e a obesidade. 
Barra de cereais com adição de farinha de resíduos de rabanete: caracterização físico-química e sensorial entre crianças

\section{CONCLUSÕES}

Um nível de adição de até $5 \%$ de farinha de resíduos de rabanete em barra de cereais é bem-aceito pelas crianças, obtendo-se aceitação sensorial similar ao produto padrão. Também, melhora o perfil nutricional do produto, aumentando os teores de minerais, proteína e fibra alimentar, além de reduzir o valor calórico. Dessa forma, a farinha de resíduos de rabanete pode ser considerada um potencial ingrediente para adição em barra de cereais e produtos similares, podendo ser oferecida ao público infantil com altas expectativas de comercialização.

\section{AGRADECIMENTOS}

As autoras agradecem aos financiadores da pesquisa, Conselho Nacional de Desenvolvimento Científico e Tecnológico (CNPq), Fundação Araucária de Apoio ao Desenvolvimento Científico e Tecnológico do Estado do Paraná (Fundação Araucária), Governo do Estado do Paraná, por intermédio da Secretaria da Ciência, Tecnologia e Ensino Superior do Estado do Paraná (SETI-PR), Programa Universidade sem Fronteiras (USF), Ministério da Saúde (MS) por meio do Departamento de Ciência e Tecnologia da Secretaria de Ciência, Tecnologia, Inovação e Insumos Estratégicos em Saúde (Decit/SCTIE/ MS) e Secretaria de Saúde do Estado do Paraná (SESA-PR), Paraná, Brasil.

\section{REFERÊNCIAS}

ASSOCIATION OF OFFICIAL ANALYTICAL COLLABORATION (AOAC) INTERNATIONAL. Official Methods of Analysis of AOAC International. 18. ed. 4 rev. Gaithersburg: AOAC, 2011.

BLIGH, E. G.; DYER, W. J. A rapid method of total lipid extraction and purification. Canadian Journal of Biochemistry and Physiology, Ottawa, v. 37, n. 8, p. 911-7, ago. 1959.

BRASIL. Ministério da Saúde. Agência Nacional de Vigilância Sanitária (ANVISA). Resolução RDC n. 54, de 12 de novembro de 2012. Regulamento técnico Mercosul sobre informação nutricional complementar (declarações de propriedades nutricionais). Diário Oficial da União, Poder Executivo, Brasília-DF, 2012. 
BRASIL. Ministério da Saúde. Agência Nacional de Vigilância Sanitária (ANVISA). Resolução RDC n. 263, de 22 de setembro de 2005. Regulamento Técnico para produtos de cereais, amidos, farinhas e farelos. Diário Oficial União, Poder Executivo, Brasília-DF, 2005.

CRISTO, T. W.; RODRIGUES, B. M.; SANTOS, N. M.; CANDIDO, C. J.; SANTOS, E. F.; NOVELLO, D. Barra de cereais com adição de farinha de casca de chuchu: caracterização físico-química e sensorial entre crianças. Semina: Ciências Biológicas e da Saúde, Londrina, v. 36, n. 2, p. 85-96, jul./dez. 2015.

DUTCOSKY, S. D. Análise sensorial de alimentos. 4. ed. Curitiba: Champagnat, 2013.

INSTITUTE OF MEDICINE (IOM). Dietary Reference Intakes for energy, carbohydrate, fiber, fat, fatty acids, cholesterol, protein and amino acids. Washington: National Academy Press, 2005.

MERRILL, A. L.; WATT, B. K. Energy values of foods: basis and derivation. Washington: United States Department of Agriculture Handbook, 1973.

TEIXEIRA, E.; MEINERT, E. M.; BARBETTA, P. A. Análise sensorial de alimentos. Florianópolis: Editora UFSC, 1987.

TEIXEIRA, F.; NUNES, G.; SANTOS, M. M. R.; CANDIDO, C. J.; SANTOS, E. F.; NOVELLO, D. Cookies adicionados de farinha da casca de beterraba: análise físico-química e sensorial entre crianças. Revista da Universidade Vale do Rio Verde, Betim, v. 15, n. 1, p. 472-88, jan./jul. 2017.

UNIVERSIDADE ESTADUAL DE CAMPINAS (UNICAMP). Tabela brasileira de composição de alimentos - TACO. 4. ed. Campinas: UNICAMP/NEPA, 2011. 